\title{
LA GESTIÓN ESTRATÉGICA EN LAS ESCUELAS PÚBLICAS EN BRASIL: CARACTERÍSTICAS Y ESPECIFICIDADES QUE INFLUYEN LAS PRÁCTICAS
}

\section{STRATEGIC MANAGEMENT IN THE BRAZILIAN PUBLIC SCHOOLS: CHARACTERISTICS AND SPECIFICITIES WHICH INFLUENCE THE PRACTICES}

\section{Pedro Henrique de Oliveira*, Marco Antonio Catussi Paschoalotto**, Ana Cláudia Fernandes Terence***, Edmundo Escrivão Filho****}

\begin{abstract}
Resumen
La administración pública tiene características y especificidades propias orientadas a brindar un servicio público de calidad considerando las necesidades y satisfacción de la población. Sin embargo, se observa que existen pocos estudios sobre la administración pública en lo que se refiere a los estudios organizacionales, especialmente en gestión escolar Así, el objetivo es identificar los factores que tienen influencia positiva en las prácticas de la administración en las escuelas. Para alcanzar el objetivo se utilizó el estudio de caso con la recopilación de datos realizada a través de entrevistas, cuestionario y grupo focal. El análisis de contenido se utilizó como forma de análisis de los datos. Los resultados apuntan que la planificación es importante para la gestión escolar, pero hay mayor tendencia en la parte pedagógica que en la administrativa y que se necesitan de herramientas específicas para la elaboración e implementación de la estrategia. Se concluye que los factores que influencian positivamente la gestión pública son la participación, la comunicación y la planificación.
\end{abstract}

Palabras clave: gestión pública; administración pública; escuela pública; gestión organizacional pública; organización pública.

\begin{abstract}
Public administration has characteristics and specificities that differentiate it from management. The quality of service provided and the satisfaction of the population are considered. However, there are few studies on public administration in organizational studies. Thus, the purpose is identifying the factors which have a positive influence on public administration practices. To reach the goal a qualitative approach was used the case study, with interviews, questionnaire, focus group, non-participant observation and field diary. Content analysis was used as a form of data analysis. The results show that planning is important for school management, but there is a tendency in the pedagogical rather than the administrative part, and specific tools are needed for the design and implementation of the strategy. We conclude that the factors that positively influence public management are participation, communication and planning at any level.
\end{abstract}

Keywords: public management; public administration; public school; public organizational management; public organization.

Recibido: 16 de septiembre de 2021 / Aceptado: 30 de diciembre de 2021

\footnotetext{
*Doutor em Engenharia de Produção, Departamento de Engenharia de Produção, Escola de Engenharia de São Carlos, Universidade de São Paulo, São Carlos, Brasil, correo: oliveiraph1@gmail.com ORCID: https://orcid.org/0000-0002-1616-6465,

**Pesquisador Pós-Doutoral, Departamento de Administração Pública e Governo, Escola de Administração de Empresas de São Paulo, Fundação Getúlio Vargas, São Paulo, Brasil, correo: marcocatussi@gmail.comORCID: https://orcid.org/0000-0003-2276-8531

***Professora Assistente, Departamento de Administração Pública, Faculdade de Ciências e Letras, Universidade Estadual Paulista "Júlio de Mesquita Filho", Araraquara, Brasil, correo: ana.terence@unesp.br ORCID: https://orcid.org/0000-0001-5657-1177,

****Professor Associado, Departamento de Engenharia de Produção, Escola de Engenharia de São Carlos, Universidade de São Paulo, São Carlos, Brasil, Correo edesfi@sc.usp.br ORCID: https://orcid.org/0000-0001-5181-5398
} 


\section{Introducción}

La cuestión de la gestión escolar surge para mejorar el rendimiento, evitar el desperdicio y mejorar el uso de los recursos para educar de manera más racional y eficiente (Paro, 2010). La visión brasileña sobre la gestión de la escuela está vinculada a una visión más política, mientras que existe una visión gerencial que coloca al director de la escuela como administrador que realiza las siguientes actividades: funciones de liderazgo; funciones administrativas; habilidades de gestión; planear actividades; dirigir recursos humanos (Lunenberg, 2010).

La planificación es una preocupación central en la gestión escolar. Se utiliza con el fin de crear condiciones para cumplir con lo establecido. Por lo tanto, la planificación describe los objetivos y caminos para que se garantice el logro de lo propuesto. La planificación escolar se entiende como derivada de la teoría general de la planificación, que busca la solución de los problemas que puedan suceder, con el establecimiento de objetivos mediante el análisis de las condiciones en las que esta planificación se desea que ocurra. Otras características de esta planificación escolar son el proceso continuo y la definición de objetivos (o compromisos de acción) (Lück, 2009). La planificación también puede entenderse como la estrategia a utilizar por organización, como en el caso del sector público o las escuelas públicas municipales.

A pesar de su importancia, no hay consenso para la definición de estrategia, apareciendo así numerosas definiciones como el plan, el pretexto, el padrón, la posición y la perspectiva (Mintzberg, 1987). Sin embargo, es necesario comprender la estrategia para comprender su naturaleza y peculiaridad frente a otros elementos organizacionales. El problema es cómo implementar la estrategia. Como posible solución existe una herramienta llamada Balanced Scorecard (BSC) con cuatro perspectivas: financieras, clientes, procesos internos, crecimiento y aprendizaje (Kaplan \& Norton, 1992). Una evolución en esta herramienta fue la creación del mapa estratégico, que es un marco visual para los objetivos en las perspectivas citadas (Kaplan \& Norton, 2000).

La administración pública en Brasil, como disciplina, está marcada por la dualidad y la existencia de un enfoque instrumental racional, es decir, guiado para ampliar la eficiencia y la eficacia, asimismo, por un enfoque político, que tiene como objetivo promover el interés público y considera las cuestiones de los valores de las personas. Se establece que las dualidades están coexistiendo, así como la ocurrencia de otras dualidades (tensiones) dentro de la administración pública: administración y política, gestión educativa (economía) y gobernanza (pedagogía).

Se puede asociar con el uso de la estrategia en el sector público dentro de la orientación racional instrumental, siendo mayor su popularidad con la introducción de la administración pública de gestión desde principios de la década de 1990 en Brasil. Se basa en los conceptos de gestión del sector privado, aunque no incluye la orientación de los beneficios, lo hace para el interés público (Andion, 2012, Pereira, 1996). Por otro lado, la administración pública corporativa parece vinculada a la orientación política, siendo una posibilidad para la gestión pública y para una mayor participación de la población (Paula, 2005).

La herramienta para la creación e implementación de la estrategia BSC logra buenos resultados en empresas privadas, siendo una posibilidad su aplicación en el sector público con el objetivo de su mejora. Esta herramienta puede ser utilizada por cualquier tipo de organización, incluyendo organizaciones no gubernamentales y unidades gubernamentales con la existencia de mapas estratégicos específicos para cada tipo de organización. Claramente, algunos puntos son diferentes cuando se trata de organizaciones privadas y organizaciones públicas que tienen una adaptación de la 
herramienta, centrándose en aumentar la satisfacción del usuario (población) o internamente para los agentes estatales (Kaplan \& Norton, 2000).

La administración pública de gestión se basa en la cultura del emprendimiento con el uso de mejores prácticas en el sector público con el objetivo de controlar, hacer procesos eficientes y generar competitividad (Paula, 2005). Un ejemplo de las buenas prácticas mencionadas es el uso de la herramienta BSC dentro del sector público. La planificación estratégica también se puede utilizar dentro de la escuela, entendida como parte del sector público. Se puede cuestionar la viabilidad de utilizar esta herramienta para una organización de este tipo.

\subsection{Problemas y objetivos}

La pregunta de investigación utilizada en este artículo es ¿¿Cuáles son los factores que influyen en la administración pública, especialmente en las escuelas públicas? El objetivo de este artículo es identificar los factores que influyen en las prácticas de la administración en las escuelas. También tiene como objetivo sistematizar las especificidades y características de la gestión pública en el uso de la gestión estratégica en las escuelas, en especial el papel de la planificación y la posibilidad de utilizar una herramienta de gestión estratégica. Está justificado que las organizaciones públicas necesitan nuevos instrumentos de gestión pública para mejorar la vida de los ciudadanos y de la sociedad. Otros factores señalados son la mejora del servicio público con mayor calidad, eficiencia, transparencia y congruente con los impuestos cobrados (Felix, Felix \& Timotéo, 2011). En esta misma línea, puede incluir la escuela un componente de la administración pública brasileña, que también necesita la creación e implementación de la estrategia con el objetivo de gestionar los riesgos de manera más eficiente y eficaz (Ministerio de la Educación y Cultura, 2006).

\section{Revisión de la literatura}

\subsection{Aspectos legales}

Comienza el debate sobre la administración pública con los aspectos jurídicos del derecho administrativo. La administración pública puede entenderse en dos direcciones: en primer lugar, el sentido subjetivo, formal y genérico, con entidades que realizan actividad administrativa, como entidades jurídicas, organismos y agentes públicos, que desempeñan la función administrativa; y, en segundo lugar, con el sentido objetivo, material y funcional, siendo la administración pública la propia función administrativa y estando predominantemente vinculada al poder ejecutivo. Hay pues, dos conclusiones: que la administración pública puede ser pasiva cuando se lleva a cabo por organizaciones públicas como una función administrativa (primer sentido) o, de lo contrario, como activa, es la propia función administrativa (segundo caso). Las consecuencias de esto es que la administración pública se puede pensar desde diferentes ángulos (Di Pietro, 2014).

Como hito inicial en el derecho administrativo, es la Constitución de la República Federativa de Brasil de 1988. La Constitución Federal, en su artículo 37, se basa en los principios de la administración pública de la siguiente manera: "La administración pública directa e indirecta de cualquiera de los Poderes de la Unión, los Estados, el Distrito Federal y los municipios cumplirá con los principios de legalidad, impersonalidad, moralidad, publicidad y eficiencia" (Constitución de la República Federativa del Brasil, 1988). 
El derecho administrativo es una rama del derecho público y se encarga de normalizar las funciones del Estado (o funciones administrativas). En Occidente, hay una doctrina que establece como tres las funciones del Estado: la legislativa, administrativa (o ejecutiva) y la judicial. Estas funciones se dividen en tres bloques, conocidos como "poderes", con el desarrollo, entonces, de lo que explican sus propios nombres: Poder Legislativo, Poder Ejecutivo y Poder Judiciario (Mello, 2010).

El artículo 37 de la Constitución Federal del Brasil restringe los principios (expresados) a sólo cinco: legalidad, impersonalidad, moralidad, publicidad y eficiencia.

Por lo tanto, tiene (Mello, 2010):

1. Principio de legalidad: la administración pública sólo puede aplicarse de conformidad con la ley;

2. Principio de propósito: busca seguir lo que está contenido en la ley, es decir, el propósito normativo, y requiere que el administrador actúe con obediencia al propósito del cargo que se le ha asignado;

3. Principio de moralidad: la administración y sus agentes deben actuar sobre principios éticos;

4. Principio de publicidad: debe de mantener plena transparencia en sus comportamientos y;

5. Principio de eficiencia: entendido como principio de buena administración, entender la forma en que opera el agente público y la forma de organizar, estructurar, la administración pública disciplinaria, con el objetivo de obtener los mejores resultados en la prestación del servicio público (Mello, 2010, Di Pietro, 2014).

Es posible dar a conocer otros puntos importantes, además de los principios constitucionales, en el artículo 37 de la Constitución Federal de 1988, como la distinción entre administración directa e indirecta y las facultades que la componen. Se entiende como administración directa los órganos o facultades a los que el Estado confiere el ejercicio de funciones administrativas, siendo, por tanto, la Unión, los Estados, el Distrito federal y los municipios (Di Pietro, 2014). A su vez, se entiende como administración indirecta "el conjunto de órganos y entidades jurídicas a los que la ley asigna el ejercicio de la función administrativa del Estado" (Di Pietro, 2014, p. 58). Por lo tanto, el desarrollo de responsabilidades se puede ver dentro de la política de educación pública con la cooperación entre las diversas entidades federativas. Se coloca la cuestión de una administración directa politizada con la necesidad de implementar la estrategia y la administración indirecta flexible, gestionando la tecnología y con una estrategia mejor definida.

La administración pública federal se forma, por un lado, por administración directa, con servicios en la estructura de la Presidencia de la República y ministerios y, por otro, componiendo administración indirecta, municipios, empresas públicas, la economía, los fundamentos y los consorcios públicos (Di Pietro, 2014).

\subsection{Administración pública internacional}

La burocracia es un término importante para entender el sentido de la administración pública. Significa una estructura compleja para las organizaciones, tanto públicas como privadas (Ketll, 2015). Otros dos significados importantes que se dan a la administración pública son el sentido de gestión de los microsistemas, o la gestión típica del sector público, con la ejecución de servicios exclusivos al sector público (Meltcalfe \& Richards, 1987), y el sentido del punto de contacto entre el Estado y la sociedad, siendo la intersección entre ellos y también un instrumento de poder (Pierre, 1995). 
Es muy difícil encontrar una definición de lo que es la administración pública, de modo que los académicos nunca han encontrado una definición común para tal área. Un problema recurrente es que, según ellos, la administración pública está experimentando una crisis de identidad (Kettl, 2015).

La evolución de la administración pública internacional tiene algunas fases: - Antecedentes de la administración pública: para esta fase, su aparición en Europa y con la asociación con la formación de Estados europeos, la legalización de nuevos aparatos políticos y administrativos y el surgimiento de la burocracia como medio de dominación racional-legal (Peci \& Cavalcanti, 2007). Los primeros estudios sobre la administración pública constituyeron una teoría política de las organizaciones, así como artículos posteriores en el campo de la administración pública (Denhardt, 2004).

- Administración pública clásica: para esta fase, y las posteriores, se tendrá en cuenta la evolución del pensamiento administrativo público estadounidense. La primera fase es la publicación de un artículo de Woodrow Wilson sobre la administración pública en 1887, llamado "El Estudio de la Administración". A partir de este trabajo se pueden destacar dos puntos principales: el primero, sobre la separación entre política y administración y el segundo, sobre la adopción de principios científicos y la búsqueda de la eficiencia (Denhardt, 2004). Por lo tanto, la administración pública clásica se caracterizaría por un enfoque gubernamental como un negocio.

- Administración pública neoclásica: la segunda fase señala algunas críticas derivadas de la concepción de la administración pública clásica. Leonard White desaprobó la separación entre la política y la administración, ya que había una interacción entre estos dos campos. Dwight Waldo, que criticó la búsqueda de la eficiencia, porque el mayor sentido de la administración pública era la prestación de servicios al público (Denhardt, 2004). Así, la administración pública neoclásica se caracterizaría precisamente por criticar al movimiento anterior y tener como objetivo principal servir al público.

- Nueva Administración Pública (NAP): la nueva administración pública surge como crítica para los dos movimientos anteriores. Esta teoría proponía que los administradores estuvieran activos en la formulación de políticas. Además, los administradores trataron de entender la ciencia basada en normas y reemplazar el énfasis en la eficiencia por el énfasis en el valor y la participación. Por lo tanto, la diferencia estaría en el objetivo central, no siendo la búsqueda de la eficiencia administrativa, sino más bien el desarrollo de estructuras más democráticas, con énfasis en la participación, los valores de la sociedad y el desarrollo del potencial humano (Denhardt, 2004).

- Gestión pública o gestión de la administración pública: Gray y Jerkins (1995) informan que este movimiento comenzó con la teoría política del papel del Estado en la vida moderna. La gestión pública viene con la idea de reinventar el gobierno, con desarrollos prácticos en todo el mundo. Algunas características son la austeridad fiscal, la mejora de la productividad de los servicios públicos, la externalización y privatización, la rendición de cuentas, la mejora del rendimiento con la reestructuración, la separación entre la política y la administración, la estrategia, la organización de los flujos a los procesos administrativos y la descentralización de la toma de decisiones (Denhardt, 2004). En Brasil, notamos un desarrollo de estas prácticas con las del Plan Maestro para la Reforma del Aparato Estatal (PDRAE) de Luiz Carlos Bresser Pereira, en 1995, en la administración pública federal.

Según Diefenbach (2009), la administración pública de gestión asume cinco puntos principales: 1) reducción de costes, reducción de la escala y privatizaciones; 2) procesos organizativos y de estructura, como la descentralización, la reorganización unitaria y la normalización y formalización de la gestión estratégica; 3) sistemas de medición y gestión del rendimiento, buscando aspectos organizativos e individuales para 
aumentar la eficiencia, productividad y calidad, con mayor rendimiento y motivación; 4) ejecución de funciones a través de gestores (ya sea en grupo o individualmente) y; 5) funcionarios y cultura corporativa, con fortalecimiento y búsqueda de satisfacer las demandas de los ciudadanos con la idea de liderar una nueva cultura corporativa.

- Nueva gestión pública o nuevo servicio público: Denhardt (2004) pone el nuevo servicio público todavía en bosquejo o como evolución. Las características del nuevo servicio público son: 1) servir a los ciudadanos, no a los consumidores; 2) dirigirse al interés público; 3) dar prioridad a la ciudadanía y al servicio público sobre el emprendimiento; 4) pensar estratégicamente y actuar democráticamente; 5) reconocer la complejidad de la rendición de cuentas;6) servir en lugar de conducir y; 7) valorar a las personas, no sólo la productividad. Para Denhardt y Denhardt (2000), este movimiento viene a reemplazar a la antigua administración pública, surgiendo en un contexto de construcción de la ciudadanía democrática, la sociedad civil y el humanismo organizacional y el discurso teórico. Como característica principal, los autores brindan el servicio y ayudan a los ciudadanos en lugar de intentar controlar y dirigir la sociedad. Merece la pena subrayar la importancia del medio ambiente para la aplicación de las características mencionadas.

Se pone en marcha la discusión de la transición de la administración pública de gestión al nuevo servicio público, siendo así un nuevo movimiento y paradigma. Se percibe la sustitución de la eficiencia por la adopción de nuevos valores públicos y la sustitución de la administración pública centrada en el mercado por la administración pública participativa, con una relación entre ciudadanos, administradores públicos y otros actores (medios de comunicación, universidades, sector privado y tercer sector) (Box et al, 2001; O'Flynn, 2007; Vigoda, 2002).

Es importante comprender aspectos de la administración pública de gestión, así como del nuevo servicio público, para comprender la organización del Estado en la actualidad (como las prácticas públicas y las políticas en materia de educación), así como las cuestiones principales en la agenda pública.

\subsection{Administración pública brasileña}

Se observa que existen numerosas teorías formales sobre la administración pública. Según Denhardt (2004), entenderlos es una forma importante de la construcción de una teoría integral e incluido en las organizaciones públicas. Entre las teorías estudiadas en la sección anterior, hay similitudes con las corrientes de la administración pública desarrolladas en Brasil.

La administración pública como disciplina está impregnada por aspectos de otros campos del conocimiento, como las ciencias jurídicas, las ciencias sociales, las ciencias políticas, la administración, las ciencias económicas. Es un área multidisciplinar con paso a un campo interdisciplinario. Andion (2012) afirma que el campo de la administración pública en el país está dominado, así como en las ciencias sociales y en la teoría de las organizaciones, por una concepción funcionalista de la ciencia.

Como resultado, se puede pensar en las corrientes de la administración pública que se han desarrollado a lo largo de los años en Brasil (Andion, 2012):

- Corriente del Estado céntrico actual: administración pública como ciencias jurídicas y ciencias administrativas. El enfoque principal se centra en la ampliación de la competencia y la racionalidad funcional en la administración pública. La organización burocrática aparece como un tipo ideal, con aspectos de jerarquía, impersonalidad, neutralidad y competencia técnica.

- Corriente pluralista: administración pública como campo político. Está marcado por el diálogo entre los campos de la administración pública y la ciencia política de aspectos 
como la descentralización y la municipalización. Avanza en el tema de la participación popular, contrayendo a la sociedad civil como un actor político importante.

- Corriente de la nueva gestión pública: administración pública como ciencia de la gestión. Es normativo, con la comprensión de la esfera pública por los principios del mercado. La nueva gestión pública sirvió de referencia para sustituir la administración pública burocrática con la administración pública de gestión (basada en la cultura del emprendimiento), para reducir el aparato estatal, el control fiscal y la aplicación de buenas prácticas del sector privado en el sector público con el fin de garantizar el control, la eficiencia y la competitividad. Pereira (2006) parte de la premisa de que la administración pública de gestión es superior a la administración pública burocrática. La cuestión gerencial puede definirse como un "conjunto de ideas y creencias que toman como máximos valores la propia gestión, el objetivo de un aumento constante de la productividad y la orientación al consumidor" (Pereira, 2006, p.20). Por último, la administración pública de gestión implica cambios en la estrategia de gestión, con ideas de descentralización y delegación de autoridad.

- Corriente del nuevo servicio público (NSP): administración pública como coproducción del bien público. Surge como una contrapropuesta de otras corrientes, basada en el nuevo modelo de gestión, teorías democráticas y de ciudadanía, modelos comunitarios y de la sociedad civil, humanismo organizacional y teoría del discurso, que destacan el diálogo a la mediación entre los diferentes actores que trabajan en la esfera pública. El ciudadano, en esta corriente, aparece como protagonista en la prestación del servicio público y en la promoción de la transformación social (Paula, 2005). La administración pública, según este enfoque, presenta una propuesta de reorganización para el aparato estatal, alternativa a la cuestión gerencial. Esta corriente difiere en algunos puntos de la corriente pluralista, como en el proyecto político, con énfasis en la participación popular; en la dimensión estructural de la gestión, con la dimensión sociopolítica; iniciativas locales de organización y gestión pública, participación en el nivel de las instituciones y en el enfoque de la gestión social (Paula, 2005).

Como conclusión, Andion (2012) afirma el predominio del paradigma funcionalista que subyace a las corrientes dominantes dentro del escenario brasileño, como la corriente del estado céntrico y la nueva gestión pública. Por otro lado, las corrientes pluralistas y la del nuevo servicio público (NSP) con otras disciplinas de las ciencias sociales, como la ciencia política, la geografía, la antropología, la sociología política, el uso de paradigmas interpretativos y humanistas radical.

Secchi (2009) propone algunos modelos organizativos y su relación con reformas administrativas para entender la evolución del pensamiento administrativo público, siendo el modelo burocrático, la cuestión gerencial, dividido en administración pública gerencial y gestión empresarial, y gobernanza pública. Aquí están las descripciones de cada uno de los modelos:

- Modelo burocrático: el modelo burocrático presenta las siguientes características: formalidad, impersonalidad y profesionalidad. Retrata aspectos centrales como la separación entre planificación y ejecución, la preocupación por la eficiencia organizativa, la equidad y la desconfianza general de la naturaleza humana.

- Modelo de la administración pública de gestión: el modelo de administración pública de gestión cierra las siguientes particularidades: productividad, orientación al servicio, descentralización, eficiencia del servicio, responsabilidad y marketization. Además, es "un modelo normativo pos-burocrático para la estructuración y la gestión de la administración pública basado en valores como la eficiencia, eficacia y competitividad" (Secchi, 2009, p. 354). 
- Modelo de gobierno emprendedor: el modelo de gobierno emprendedor aporta los siguientes aspectos: productividad, orientación a los servicios, descentralización, eficiencia en la prestación de servicios, accountability y marketization, así como el modelo anterior, ambos denominados gerencial. Osborne y Gaebler en 1992 inauguran este modelo, con el libro "Reinventando el gobierno". Los autores prescriben diez tipos de gobierno para una organización pública racional y eficaz: gobierno catalizador, gobierno de propiedad comunitaria, gobierno competitivo, gobierno orientado a misiones, resultados orientados al gobierno, gobierno orientado al cliente, gobierno empresarial, gobierno preventivo, gobierno descentralizado, gobierno orientado al mercado.

- Modelo de gobernanza pública: el modelo de gobernanza pública tiene las siguientes características: relación entre actores públicos y privados en el proceso de desarrollo de políticas públicas, pluralismo en la participación de actores para la formulación e implementación de políticas públicas, cambio en el papel del Estado, menos jerárquico y menos monopolístico, reducción de criterios técnicos en los procesos de toma de decisiones y refuerzo de los mecanismos participativos de deliberación en la esfera pública, participación para la construcción de políticas públicas utilizando la democracia deliberativa y una red de políticas públicas y la coordinación de los actores estatales y no estatales en las operaciones gubernamentales.

\section{Método}

El método de investigación utilizado envuelve un paradigma de investigación interpretativo. El enfoque utilizado fue cualitativo adaptándose a los objetivos de investigación. Este enfoque busca entender la relación entre el mundo objetivo y la subjetividad del sujeto, que no puede traducirse en números, además de interpretar los fenómenos y la atribución de significados (Silva \& Menezes, 2005). El enfoque cualitativo se basa en la fenomenología como un paradigma más influyente, buscando entender la dinámica del ser humano, además de utilizar la observación e interpretación de la información categorizada para analizar los datos (Turato, 2005). Solo uno de los pasos de la investigación tuvo un enfoque cuantitativo, como se describe a continuación.

Para alcanzar el objetivo propuesto, se utilizó el estudio de caso "cuando se trata del estudio profundo y exhaustivo de uno o algunos objetos de una manera que permita su amplio y detallado conocimiento" (Silva \& Menezes, 2005, p.21). Además, la investigación se aplicó en relación con el problema de la investigación, mediante la generación de conocimientos para la aplicación práctica y dirigido a resolver problemas específicos (Silva \& Menezes, 2005). La obra es descriptiva, porque representa las características de una población determinada (Gil, 2002).

El sistema escolar brasileño se divide en educación básica, con los siguientes niveles: educación infantil, primaria y secundaria; y educación superior. La educación infantil es responsabilidad de los municipios, mientras que la educación secundaria es responsabilidad de los Estados. La educación primaria se comparte entre municipios y Estados. Finalmente, la educación superior se divide principalmente entre los Estados y el gobierno federal. En todos los niveles también hay participación del sector privado (Ley del Lineamentos y Bases de la Educación Nacional Brasileña, 1996). Así, se realizó una investigación en escuelas primarias públicas entre los años 2016 y 2019.

La investigación tuvo un primer paso con el uso de datos cuantitativos sobre el rendimiento escolar. El insumo se generó de la inversión anual por estudiante, nivel socioeconómico (NSE) e infraestructura. La producción se formó por el rendimiento en el Índice Básico de Desarrollo Escolar (IDEB). La división de escuelas siguió tres grupos, que van desde el rendimiento más bajo hasta el más alto, el grupo 2 y 3 ; grupo 4 y el 
grupo 5 y 6. El último grupo, el rango 5 y 6 fue elegido, porque fue el único que presentó más de 20 escuelas en el Estado de São Paulo, posicionadas como la necesidad de investigación en cuestión por el mayor muestreo de escuelas.

Se tomó la decisión arbitrariamente de elegir la ciudad de Ribeirão Preto/SP para la investigación de escuelas públicas. Se contactó con el Secretario de Educación del municipio para obtener los datos y el inicio de la investigación. Las opciones se dividieron en dos grupos, uno de mejor desempeño (Escuela A, Escuela B, Escuela C y Escuela D) y uno de los peores rendimientos (Escuela E, Escuela F, Escuela G y Escuela H). Vale la pena señalar que estas escuelas pertenecen al mismo grupo socioeconómico.

Las categorías utilizadas en este artículo fueron la planificación, un proceso de pasos y acciones que guían a la organización para que los objetivos y metas se alcancen dentro del entorno y sean elegidos con satisfacción, y las especificidades de la gestión pública, entendidas por las características particulares de la gestión pública para que la organización logre sus objetivos, como el seguimiento de las leyes, el entorno político y no buscar beneficios, sino la calidad de los servicios para la sociedad. En esta investigación, la técnica de recopilación de datos fue realizada por el cuestionario, el grupo de enfoque y la entrevista.

La técnica de recopilación de datos de entrevistas se entiende por "obtener información de un entrevistado, sobre un tema o problema determinado". (Silva \& Menezes, 2005, p. 33). En la investigación, se utilizaron dos guiones de entrevistas, uno durante las entrevistas y el otro después del uso del grupo de enfoque. Estos scripts tenían preguntas abiertas y múltiples opciones para recopilar datos de información relevante. Cabe destacar que los guiones de la entrevista fueron pasados por una prueba previa a sus aplicaciones. Se realizaron ocho entrevistas semiestructuradas con cada director de la escuela. La grabadora de voz fue utilizada para las entrevistas, con la excepción de los directores de las escuelas $\mathrm{E}$ y $\mathrm{F}$ que no permitían su uso.

El grupo de enfoque se utiliza como un formulario de entrevista para grupos pequeños en el intento de aprender sobre la biografía y las estructuras de vida de los participantes del grupo. Se coloca como un punto principal particular o de relevancia para el investigador y el grupo. El investigador del grupo de enfoque tiene el papel de moderador (Breg, 1998). En este artículo esta técnica se utilizó para obtener información en cuatro reuniones posteriores con los directores de las escuelas. Los 08 directores fueron invitados a esta etapa, pero sólo tres asistieron (Escuela B, Escuela C y Escuela G). Estas reuniones duraron tres horas y media cada una, abordando los temas de la revisión literaria de este estudio.

El cuestionario, utilizado en el paso cuantitativo, es una "técnica estructurada para la recopilación de datos en una serie de preguntas, escritas u orales, que un entrevistado debe responder". El objetivo es transformar la información deseada en preguntas para motivar al entrevistado en las respuestas para completar el guion (Malhotra, 2006, pp.290-291). En la etapa de grupos focales, se utilizó un cuestionario para conocer la percepción y la reacción de los directores de la escuela sobre la etapa aplicada.

Por último, la técnica de análisis de datos utilizada en la investigación fue el análisis de contenido. Es definida como "un conjunto de instrumentos tecnológicos cada vez más sutiles en constante mejora, que se aplican a discursos extremadamente diversos (contenido y continentes)". Se utilizó para el tratamiento, inferencia e interpretación de los datos, que se organizan por las siguientes etapas: preanálisis, exploración del material y el tratamiento de los resultados obtenidos e interpretación (Bardin, 1977, p.9). 


\section{Resultados y discusiones}

La sección de resultados y discusiones comienza a presentar el año de fundación de las 08 escuelas encuestadas en este artículo.

Tabla 2 - Escuelas y año de fundación

\begin{tabular}{|c|c|}
\hline Escuela & Año de Fundación \\
\hline Escuela A & 1960 \\
\hline Escuela B & 1993 \\
\hline Escuela C & 2000 \\
\hline Escuela D & 1998 \\
\hline Escuela E & No había datos \\
\hline Escuela F & 1996 \\
\hline Escuela G & 1978 \\
\hline Escuela H & 1995 \\
\hline
\end{tabular}

Fuente: Ayuntamiento de Ribeirão Preto (2016).

En la comprensión de la administración pública (Di Pietro, 2014), se puede enmarcar la escuela pública municipal como una unidad de ejecución en el sentido subjetivo, formal y genérico, realizando la función administrativa. El Departamento Municipal de Educación de Ribeirão Preto/SP encaja en el segundo sentido, es decir, objetivo, material y funcional, siendo la propia función administrativa. Dicha Secretaría se entiende como una función administrativa (o ejecutiva), es decir, el Poder Ejecutivo.

Los principios de la administración pública (caput del artículo 37 de la Constitución Federal, Mello, 2010, Di Pietro, 2014) aparecen en el desarrollo de actividades de la escuela pública, como el principio de legalidad al utilizar las leyes; principio de propósito, siguiendo lo contenido en la ley; principio de moralidad, actuando de acuerdo con los principios éticos. Sin embargo, se examinan dos principios, la publicidad, de mantener la transparencia de los actos y la eficiencia, entendidas como una buena administración. No fue posible obtener los Proyectos Políticos Pedagógicos (PPP) de las entrevistas escolares, y la sugerencia para el Departamento Municipal de Educación fue la provisión de PPP para el análisis de quien esté interesado. Este hecho está claramente relacionado con el principio de la publicidad. Las escuelas tenían recursos financieros prácticamente iguales, pero diferentes resultados en el IDEB. Uno puede asociarse con el principio de eficiencia, pensando en cómo organizar la escuela pública para obtener los mejores resultados en la prestación de servicio público (una educación de mejor calidad).

Contrariamente a lo expresado en la literatura, las escuelas tenían prácticas similares de gestión escolar interna. Sin embargo, como lo expresaron los académicos en general, la actuación fue heterogénea (Galilea \& Saavedra, 2013).

La administración pública necesita que sea imparcial, sostenible, eficiente, transparente, eficaz, legítima y saludable. De ahí la importancia de analizar la calidad de la administración pública y promover las políticas públicas para el fortalecimiento institucional de la administración pública (Ruiz, 2012). En este sentido, es importante analizar el análisis organizacional público en América Latina, así como el análisis de políticas educativas públicas más efectivas.

La distinción entre administración directa e indirecta (Pietro, 2014) también se aplica a la escuela pública. Esto se entiende como administración directa mediante la prestación de servicios públicos por parte del Estado. Claramente, la relación de las escuelas era fuerte con el municipio, porque estaba vinculado a él. Se nota el desarrollo de responsabilidades en el mantenimiento de Fondo para el Mantenimiento y Desarrollo de la Educación Básica y Valorización de los Profesionales de la Educación (FUNDEB). 
La Escuela C dijo que estaba relacionada con las tres entidades federativas (Municipio, Estado y Unión), siendo una sola para reportar la relación con el Estado de Sao Paulo. La escuela E dijo que la relación era fuerte con el municipio porque estaba vinculada a ella. Además, señaló la transferencia de recursos financieros por parte del municipio (subvención) y el gobierno federal (Programa de Dinero Directo en la Escuela).

La relación de la transferencia de recursos financieros específicos de la escuela ocurrió con la participación del gobierno municipal por medio de subvenciones y a través del gobierno federal con ayuda del Programa de Dinero Directo en Las Escuelas (PDDE).

La escuela pública municipal está comprendida en lo que es conocido, en la administración pública, por burocracia pública (Kettl, 2015), conforme a lo citado por los directores para la organización de trabajo; gestión de microsistemas, típicos del sector público, con la ejecución de servicios del sector público (Meltcalfe \& Richards,1987) y; el punto de contacto entre el Estado y la sociedad (Pierre, 1995), en el que la educación aparece como una gestión típica del sector público y la interacción entre el Estado (realizada por el municipio) y la sociedad (padres de estudiantes y estudiantes).

No hubo estandarización de las actividades por parte de los directores de las escuelas. Básicamente, el trabajo diario se describió mediante la entrada en la escuela y la supervisión del edificio, el seguimiento de la entrada de estudiantes y empleados, el servicio comunitario, el apoyo a las actividades pedagógicas y administrativas.

Tampoco se ha normalizado la descripción de las actividades administrativas y pedagógicas. Una confusión fue percibida en la distinción de las dos funciones por los directores de la escuela, porque, en la vida cotidiana, es muy difícil distinguirlas. Las actividades administrativas mencionadas fueron las más diversas, como parte procedimental de la escuela, resolución de problemas, apoyo a profesores y estudiantes, manejo de recursos financieros, representación de la escuela (Consejo Escolar, reuniones con maestros, con la Asociación de Padres y Maestros, con el Departamento Municipal de Educación), pago de maestros y rendición de cuentas. También se percibió cierta prioridad con las actividades pedagógicas. Al igual que con las actividades administrativas, las actividades pedagógicas fueron señaladas como las más diversas por los directores de la escuela, incluyendo: apoyo a los maestros y estudiantes que asisten a los estudiantes para hablar sobre su comportamiento, visitas comunitarias (padres de estudiantes) y seguimiento de la ejecución de las actividades del Proyecto Político Pedagógico (PPP).

Según los directores, el uso de los recursos financieros disponibles se definió a través del Consejo Escolar, con la representación del equipo directivo (gestión), profesores, empleados, padres y estudiantes. La Asociación de Padres y Maestros (APM) tuvo una función que supervisa los gastos realizados por la escuela pública municipal.

La mayoría de los directores señalaron la legislación aplicada a la escuela como razonable. Algunos de ellos han puesto la legislación en su forma insuficiente o inadecuada. Citaron la necesidad de revisar algunas leyes, como la ley de accesibilidad para personas con necesidades especiales y sobre una mayor autonomía del maestro en el aula.

Según los directores, los mecanismos de participación en las decisiones de la escuela eran reuniones del Consejo Escolar. También citaron reuniones con maestros, la Asociación de Padres y Maestros (APM) y padres de los estudiantes (comunidad).

No hubo consenso sobre las principales diferencias entre la escuela pública y la escuela privada. Los directores entrevistados citaron las siguientes diferencias: mayor estabilidad por parte de la escuela pública con maestros y empleados, debido a la entrada por licitación pública y centrarse en el aprendizaje y el contexto social del estudiante. En cuanto a la diferencia en la gestión de los dos tipos de escuelas mencionadas, la escuela 
privada defendió los intereses sólo del grupo de gestión (propietarios), mientras que la escuela pública asistió a la comunidad local (vecindario donde está localizado). No se señalaron las diferencias entre la administración de empresas y la administración pública, como las organizaciones públicas más burocráticas, la menor acción de orientación para el mercado ( $\sin$ ánimo de lucro) es menos compromiso con los aspectos organizacionales (Boyne, 2002).

La importancia de las demandas locales, aspectos éticos y culturales, tradiciones religiosas de la región en la que se discute el servicio de educación pública, ya que puede diferenciarse como por ejemplo en América Latina e incluso en las regiones brasileñas (Galilea \& Saavedra, 2013). Por lo tanto, se debe tener cuidado al generar los análisis y discusiones.

En el grupo de enfoque, se discutió la composición del equipo directivo. El entendimiento era que el equipo directivo debía estar compuesto por un director general, un coordinador administrativo y un coordinador pedagógico. Los directores que participan en el grupo focal señalaron que el equipo directivo aprende en la práctica, porque el curso de Pedagogía no ofrece subvenciones para la gestión escolar.

La propuesta de la BSC fue colocada como el plan administrativo exclusivo de la parte administrativa, incluyendo la gestión estratégica, debido a la falta de planes escolares. Por lo tanto, es necesario que la propuesta de aplicación del BSC se adhiera a la realidad de la escuela pública, respetando las particularidades de cada unidad escolar. Los directores señalaron que las escuelas realmente necesitan mecanismos para fortalecer el aprendizaje del cuerpo estudiantil. Así, el BSC se utilizaría como evaluación de la organización en su conjunto, tanto en los aspectos pedagógicos como administrativos. Poco a poco, podría ser utilizado por todas las escuelas públicas municipales del sistema escolar Ribeirão Preto/SP.

Los beneficios de utilizar la gestión estratégica en el sector público fueron la alineación en la gestión, la mirada a largo plazo, que aporta mejoras a la gestión estratégica. También ponen el beneficio de la visión holística de la escuela.

Los factores de restringir el uso de la gestión estratégica en el sector público fueron la falta de recursos financieros, factores políticos, personas equivocadas en los puestos errados, legislación (leyes antiguas), falta de personal técnico, falta de profesionalismo y la cuestión por factor político, sin tener en cuenta los factores de conocimientos técnicos. Citaron problemas en el calendario escolar y un director citó un caso de problema con un maestro, que culminó en la eliminación de este.

Las especificidades de la gestión pública para el uso de la gestión estratégica fueron el enfoque en la satisfacción de la sociedad, objetivos más orientados al público, mientras que debe contemplar ambos aspectos administrativos también. Otras especificidades abordadas fueron la cuestión de la escuela pública, dirigida a la población con el uso de los recursos públicos y la cuestión de la participación y colaboración de la comunidad escolar para la toma de decisiones. Destacaron la necesidad de aumentar la duración de los mandatos en el Consejo Escolar, la Asociación de Padres y Maestros, además, también mencionaron la realización de la proyección del aula para el año posterior (2017). En esta etapa, también se aplicó un cuestionario para evaluar la reacción de los directores en el grupo de enfoque. En general, el grupo de enfoque fue bien evaluado por los directores de las escuelas participantes.

\section{Conclusiones}

El objetivo de este artículo es identificar los factores que tienen una influencia en la administración pública. Los factores que tienen una influencia en la gestión pública 
fueron la participación, con el establecimiento de mecanismos de decisión compartidos entre la comunidad escolar y la unidad de gestión; comunicación, la transferencia de información de manera integrada y rápida por parte de la escuela y; la estrategia, que en este caso era un factor limitante para el trabajo escolar y con el uso de una herramienta para la gestión estratégica podría mejorar las actividades de eficiencia y eficacia.

Con respecto a las especificidades de la gestión pública en el uso de la gestión estratégica son la satisfacción de la sociedad, los objetivos orientados al público (no generar beneficios), el uso de los recursos públicos, la participación y la colaboración para la toma de decisiones. El papel de la planificación es importante en la gestión escolar. Sin embargo, se percibe un enfoque en la cuestión pedagógica, con la falta de un plan específico para la parte administrativa. La elaboración de este plan podría ser resuelto por las herramientas de BSC y el mapa estratégico. Se observa que los directores tuvieron dificultades para implementar la planificación, como en el caso de los problemas mencionados en el calendario escolar. En consecuencia, la posibilidad de utilizar herramientas vitales específicas de gestión se coloca como positiva para una mejor elaboración e implementación de la estrategia y la planificación. Las principales actividades desarrolladas en la gestión escolar por la dirección fueron en relación con las conversaciones con los estudiantes y las visitas a la comunidad externa. Una vez más, se percibe el enfoque pedagógico y la discapacidad en el servicio de funciones administrativas en la gestión pública.

Sobre los mecanismos de planificación de la escuela pública municipal destaca el Proyecto Político Pedagógico (PPP), el plan escolar, el plan de lecciones y la planificación participativa anual. Se percibe que contradictoriamente como fue expuesto en la literatura, la PDE no tiene una función de planificación en la escuela, siendo sólo un medio de transferir dinero a una solución tópica. La PPP asume tanto la función política como pedagógica de la escuela pública municipal. El plan escolar tiene un mayor alcance en contenido y temporalidad. El plan de lecciones es el plan específico del maestro para la vida cotidiana en el salón de clases. La planificación participativa anual se realiza internamente, en la que se discute, entre otras cosas, el calendario y las actividades a lo largo de los dos semestres.

Se percibe la importancia de cada fase de la investigación para obtener los resultados y alcanzar los objetivos. La fase de entrevistas por la descripción de las escuelas y la gestión pública en la práctica.

Básicamente, se pueden sacar algunas conclusiones sobre las escuelas públicas municipales investigadas:

- Las especificidades son el uso de leyes, factores políticos, indicación de personas para puestos comisionados y;

- Las sugerencias de mejora en la gestión pública de las escuelas, estaban disponibles de los planes escolares en el sitio web de la Secretaría de Educación, la disponibilidad de documentos escolares en los portales de cada institución; el uso de la firma digital por parte de empleados y maestros, estipulan horas de atención, la creación de un banco de buenas prácticas para el intercambio de conocimientos y materiales de la red escolar municipal de Ribeirão Preto/SP; capacitaciones centradas en la discusión e intercambio de conocimientos entre la red escolar municipal de Ribeirão Preto/SP; la formación centrada en la discusión e intercambio de conocimientos entre la empleados de las escuelas públicas municipales y no sólo para la resolución de problemas por parte del Departamento Municipal de Educación; uso de BSC y mapa estratégico como plan administrativo de la escuela y; 
- Uso del BSC y mapa estratégico en la red de educación municipal y el Departamento Municipal de Educación como alineación administrativa.

La limitación de este estudio fue la baja participación de los directores de la escuela en la fase de grupo focal y el uso del guión de entrevista semiestructurado que puede estandarizar las declaraciones de los directores de la escuela. La subjetividad del investigador se coloca como un factor importante para el análisis y las discusiones de los resultados. Como sugerencia de resultados, se coloca el análisis de las funciones y el papel que cumplen los directores de escuelas y las políticas de educación pública para la mejora de la educación pública brasileña. Por lo tanto, con la realización de esta investigación, se espera que haya contribuido al avance del conocimiento en esta área y que permita el desarrollo de nuevas investigaciones relacionadas con la gestión pública. 


\section{Referencias}

Andion, C. (2012). Por una nueva interpretación de los cambios de paradigma en la administración pública. Cadernos EBAPE.BR, 10(1), 1 a 19. https://bibliotecadigital.fgv.br/ojs/index.php/cadernosebape/article/view/5243

Bardin, L. (1977). Análise de conteúdo. Lisboa: Edições70

Box, R.C., Marshall, G.S., Reed, B., \& Reed, C.M. (2001), New Public Management and Substantive Democracy. Public Administration Review, 61: 608-619. https://doi.org/10.1111/0033-3352.00131

Boyne, G.A. (2002), Public and Private Management: What's the Difference?. Journal of Management Studies, 39: 97-122. https://doi.org/10.1111/1467-6486.00284

Breg, B. L. (2007). Qualitative research methods for the social science. California State University, Long Beach.

Bresser-Pereira, L. C. (1996). Administração pública gerencial: estratégia e estrutura para um novo Estado (Vol. 9). Brasília: ENAP.

Constitución de la República Federativa del Brasil (1988). http://www.planalto.gov.br/ccivil 03/constituicao/constituicao.htm

Denhardt, R. B. \&Denhardt, J. V. (2000). The new public service: serving rather than steering., $\quad$ Public administration review, 60(6): $\quad$ 549-559 https://doi.org/10.1111/0033-3352.00117.

Denhardt, R. B. (2004). Teoria geral da administração pública. São Paulo: Thomson.

Di Pietro, M. S. Z. (2014). Direito administrativo, (27a Ed.) São Paulo: Atlas.

Diefenbach, T. (2009). New public management in public sector organizations: the dark sides of managerialistic 'enlightenment', Public administration, 87(4): 892-909 https://doi.org/10.1111/j.1467-9299.2009.01766.x

Fayol, H. (1975). Administração industrial e geral. São Paulo: Atlas.

Felix, R., Felix, P. P. \& Timóteo, R. (2011). Balanced Scorecard: adequação para a gestão estratégica nas organizações públicas, Revista do Serviço Público, 62(1): 51-74. https://doi.org/10.21874/rsp.v62i1.61

Galilea O.,S., \& Saavedra, L. L. (2013). El estado de los servicios descentralizados en América Latina. Una perspectiva comparada, Revista del CLAD Reforma y Democracia, 55:17-48. https://www.redalyc.org/pdf/3575/357533687002.pdf

Gil, A. C. (2002). Como elaborar projetos de pesquisa. São Paulo: Atlas.

Gray, A., \& Jerkins, B. (2006). From public administration to public management: reassessing a revolution?, Comparative Public Administration. Emerald Group Publishing Limited, 543-572. https://doi.org/10.1111/j.1467-9299.1995.tb00818.x

Kaplan, R. S., \& Norton, D. P. (1992)., The Balanced scorecard: measures that drive performance, Harvard Business Review, 70(1), pp. 71-79.

Kaplan, R. S., \& Norton, D. P. (1996). Linking the Balanced Scorecard to Strategy, California Management Review, 39(1) https://doi.org/10.2307\%2F41165876

Kettl, D. F. (2015). The transformation of governance: Public administration for the twenty-first century. JHU Press.

Ley del Lineamentos y Bases de la Educación Nacional Brasileña (1996). http://www.planalto.gov.br/ccivil_03/leis/19394.htm

Lück, H. (2009). Dimensões de gestão escolar e suas competências.,Curitiba: Editora Positivo.

Lunenburg, F. C. (2010). The principal and the school: What do principals do?, National Forum of Educational Administration \& Supervision Journal. 27 ( 4).

Malhotra, N. K. (2006). Pesquisa de marketing: uma orientação aplicada. Porto Alegre: Bookman Editora. 
Mello, C. A. B. (2010). Curso de direito administrativo., 27.ed. São Paulo: Malheiros.

Metcalfe, L., \& Richards, S. (1987). Evolving public management cultures., Managing Public Organizations. London: Sage: 65-86.

Ministerio de la Educación y Cultura (2006). Como elaborar o plano de desenvolvimento da escola. Brasília: ftp://ftp.fnde.gov.br/web/fundescola/publicacoes_manuais_tecnicos/pde_escola.pd $\underline{f}$

Mintzberg, H. (1973). The Nature of managerial work. New York: Harper and Row.

Mintzberg, H. (1987). The strategy concept I: Five Ps for strategy., California management review 30(1): 11-24. https://doi.org/10.2307\%2F41165263

O'Flynn, J. (2007). From new public management to public value: Paradigmatic change and managerial implications., Australian journal of public administration 66(3): 353-366. https://doi.org/10.1111/j.1467-8500.2007.00545.x

Paro, V. H. (2010). A educação, a política e a administração: reflexões sobre a prática do diretor de escola., Educação e Pesquisa 36(3). https://doi.org/10.1590/S151797022010000300008

Paula, A. P. P. (2005), Administração pública brasileira entre o gerencialismo e a gestão social., RAE-revista de administração de empresas, 45(1):36-49. http://dx.doi.org/10.1590/S0034-75902005000100005

Peci, A.\&, y Bianor S. C. (2007). Administração pública e seu ensino: um campo em busca de legitimação, Revista do Serviço Público, (58). https://revista.enap.gov.br/index.php/RSP/article/view/5236

Pierre, J., ed. (1995) Bureaucracy in the modern state: an introduction to comparative public administration. Edward Elgar Publishing.

Prefeitura municipal de Ribeirão Preto (2016). Secretaria Municipal de Educação. Obtenido enhttps://www.ribeiraopreto.sp.gov.br/seducacao/i15principal.php\#\&panel1-2

Ruiz L., \& Joaquín S. (2012). Calidad en la gestión pública: del azar a la necesidad, Revista del CLAD Reforma y Democracia: 54(1): 63-94.

Secchi, L. (2009), Modelos organizacionais e reformas da administração pública., Revista de Administração Pública, 43(2): 347-369. https://doi.org/10.1590/S003476122009000200004

Silva, E. L. \& Menezes, E. M. (2001). Metodologia da pesquisa e elaboração de dissertação. UFSC, Florianopólis.

Turato, E. R. (2005). Métodos qualitativos e quantitativos na área da saúde: definições, diferenças e seus objetos de pesquisa,Revista de Saúde pública 39(3):507-514. https://doi.org/10.1590/S0034-89102005000300025

Vigoda, E. (2002). From responsiveness to collaboration: Governance, citizens, and the next generation of public administration, Public administration review 62(5): 527540. https://doi.org/10.1111/1540-6210.00235 